\title{
Tissue expression and antibacterial activity of host defense peptides in chicken
}

Mi Ok Lee ${ }^{1,2 \dagger}$, Hyun-Jun Jang ${ }^{1,3 \dagger}$, Deivendran Rengaraj ${ }^{1}$, Seo-Yeong Yang ${ }^{1}$, Jae Yong Han ${ }^{1}$, Susan J. Lamont ${ }^{4}$ and James E. Womack ${ }^{1,2^{*}}$

\begin{abstract}
Background: Host defence peptides are a diverse group of small, cationic peptides and are important elements of the first line of defense against pathogens in animals. Expression and functional analysis of host defense peptides has been evaluated in chicken but there are no direct, comprehensive comparisons with all gene family and individual genes.

Results: We examined the expression patterns of all known cathelicidins, $\beta$-defensins and NK-lysin in multiple selected tissues from chickens. CATH1 through 3 were predominantly expressed in the bone marrow, whereas CATHB1 was predominant in bursa of Fabricius. The tissue specific pattern of $\beta$-defensins generally fell into two groups. $\beta$-defensin 1-7 expression was predominantly in bone marrow, whereas $\beta$-defensin8-10 and $\beta$-defensin 13 were highly expressed in liver. NK-lysin expression was highest in spleen. We synthesized peptide products of these gene families and analysed their antibacterial efficacy. Most of the host defense peptides showed antibacterial activity against E.coli with dose-dependent efficacy. $\beta$-defensin 4 and CATH3 displayed the strongest antibacterial activity among all tested chicken HDPs. Microscopic analyses revealed the killing of bacterium by disrupting membranes with peptide treatment.
\end{abstract}

Conclusions: These results demonstrate dose-dependent antimicrobial effects of chicken HDPs mediated by membrane damage and demonstrate the differential tissue expression pattern of bioactive HDPs in chicken and the relative antimicrobial potency of the peptides they encode.

Keywords: Host defence peptides (HDPs), Antimicrobial, Chicken

\section{Background}

Bacterial infections in chickens are important not only for the health and productivity of the animals but also as a reservoir of foodborne human pathogens such as Salmonella enterica. Innate immunity is important in controlling bacterial infection, particularly at mucosal surfaces such as the gastrointestinsal, respiratory and reproductive tracts. Innate immune agents include antimicrobial secretions such as lysozyme, mucocilliary clearance, the acid environment of the gizzard

\footnotetext{
* Correspondence: jwomack@crm.tamu.edu

${ }^{\dagger}$ Equal contributors

'WCU Biomodulation Major, Department of Agricultural Biotechnology, Seoul National University, Seoul, Korea

2Department of Veterinary Pathobiology, Texas A \& M University, College

Station, TX, USA

Full list of author information is available at the end of the article
}

and proventriculus and tight cellular junction at epithelial layers [1].

Host defense peptides (HDPs) are a diverse group of small, cationic peptides present in A wide variety of organisms including both animals and plants [2-6]. HDPs are an important first line of defense, particularly in those species whose adaptive immune system is lacking or primitive. A majority of HDPs are strategically synthesized in the host phagocytic and mucosal epithelial cells that regularly encounter microorganisms from the environment. Mature HDPs are broadly active against Gram-negative and Gram-positive bacteria, mycobacteria, fungi, viruses and even cancerous cells [7-9]. Several classification schemes have been proposed for AMPs; however, most AMPs are generally categorized into four clusters based on their secondary structures: peptides with a linear $\alpha$-helical structure [10-12], cyclic peptides with a 
$\beta$-sheet structure [13-17], peptides with a $\beta$-hairpin structure [18], and peptides with a linear structure $[19,20]$.

It has become clear that HDPs are important and significant components of host defence against infection. The killing of bacteria appears to be very fast, ranging from 10 to $30 \mathrm{~min}$ for killing of S. enteritidis [21] and 30-60 min for killing of E.coli [22]. We have demonstrated chicken NK-lysin to destroy E. coli cell membranes within 5 min [23]. Furthermore, HDPs kill bacteria primarily through physical electrostatic interactions and membrane disruption. Therefore, it is difficult for microbes to gain resistance to HDPs [7, 24]. At the same time, most HDP's have the capacity to recruit and activate immune cells and facilitate the resolution of inflammation $[24,25]$. Therefore, it is not easy to differentiate therapeutic potential of HDPs, particularly against antibiotic-resistance bacteria. A highly promising approach to overcome drug resistance is to explore and exploit the huge diversity of innovative bioactive-engineered molecules provided by nature to fight pathogens. These include HDPs, natural products involved in the defense systems. So, with their obvious potential as novel therapeutic agents, understanding the HDPs, including the relationships between structure and mode of action of these molecules, is essential for the development of novel peptide-based antibiotics and immunotherapeutic tools.

Three major groups of HDPs, namely cathelicidins (CATH), defensins and NK-lysin are present in vertebrate animals. Defensins constitute a large family of small, cysteine rich, cationic peptides that are capable of killing a broad spectrum of pathogens [26-29]. Vertebrate defensins are classified into three subfamilies, the $\alpha-, \beta-$, and $\theta$-defensins, characterized by different spacing of the six conserved cysteines. Cathelicidins are recognized by the presence of cathelin-like domains. The signal peptide and cathelin-like domains are well conserved across species, but the mature peptide sequences at the C-terminal regions are highly diverse [30]. Whereas the defensin structure is based on a common $\beta$-sheet core stabilized by three disulfide bonds [2], CATH s are highly heterogeneous. NK-lysin is a member of the saposin-like protein (SALIP) family, and is orthologous with human granulysin with a $\alpha$-helical structure [9].

The first avian HDPs discovered were $\beta$-defensins from chicken and turkey, reported in the mid 1990-'s [31], and increasing information about HDPs in other avians species is becoming available [32]. The sequencing of the chicken (Gallus gallus) genome revealed the presence of a cluster of 14 different genes on chromosome 3 coding for avian defensins ( $A v B D$ ) and designated then as $A v B D 1$ to $-14[33,34]$ and $4 C A T H s$ densely clustered at the proximal end of chromosome $2[35,36]$. NK-lysin was recently mapped to the distal end of chromosome 22 [37].
The highly inbred Leghorn Ghs- 6 line has been used in many studies of immune function, including serving as a parental line of an advanced intercross line used to identify the association of genetic variants in the $A v B D$ gene cluster with colonization of the cecum with Salmonella enterica serovar Enteritidis [38]. The bursa of Fabricius, a specialized immune organ in birds, arises from bursal epithelial cells around embryonic day 4, reaches a maximum size at 6-12 weeks after hatching [39] and previously demonstrated high expression of several of AvBDs [34]. The gene expression and antibacterial efficacy of all four $C A T H$ and several $A v B D s$ has been evaluated individually, but there are no reports comparing the full spectrum of tissue expression and antimicrobial activity of chicken HDPs concordantly. Here, we have examined expression patterns of $14 A v B D, 4 C A T H$ and NK-lysin with the highly inbred Leghorn Ghs-6 line and compared the antimicrobial activity of the peptides encoded against E.coli. Morphological change of E.coli membranes by $C A T H$ peptide treatment was also examined.

\section{Methods \\ Birds}

Chicks of the highly inbred Leghorn Ghs-6 line were produced and maintained in the Poultry Genetics Program at Iowa State University (Ames, IA). Birds were raised in lightand temperature-controlled pens with wood-shaving bedding and continual access to water and food meeting all NRC nutritional requirements. At 7 weeks of age, birds were euthanized according to the approved Institutional Animal Care and Use Committee protocol (Log \#4-03-5425-G) and tissues immediately dissected. Bursa of Fabricius, thymus, spleen, bone marrow, cecal tonsil, duodenal loop, and liver tissue were collected. Samples consisting of either the entire tissue, or sections totalling approximately 1.0 cubic $\mathrm{cm}$ from larger tissues were harvested. The cecal tonsil included the lymphoid aggregates and surrounding tissue at the intersection of the two ceca and the gastrointestinal tract. Bone marrow was collected by expressing the marrow from both tibias of each bird with a narrow sterile wooden rod. Tissues were placed into RNAlater until used for isolation of mRNA.

\section{RNA extraction and quantitative reverse-transcription polymerase chain reaction (RT-PCR)}

RNA extraction was performed using RNeasy Mini Kit (Qiagen) according to the manufacturer's instructions. Total RNA samples were extracted from 5 birds tissues and used as a template for reverse transcription. cDNA was obtained by reverse transcriptase SuperScript ${ }^{\odot}$ III First-Strand Synthesis System using $2 \mu \mathrm{g}$ total RNA. The relative abundance of mRNA from genes was assessed by real time reverse-transcription (RT)-PCR using a Lightcycler 480 (Bio-Rad) and a Lightcycler 480 SYBR Green I master (Bio- 
Rad). Primer pairs specific for the amplification of $A v B D$, cathelicidin and NK-lysin genes are shown in Additional file 1: Table S1. PCR products were subjected to melt curve analysis and sequenced to confirm amplification of the correct gene. Data were analyzed by ddCt method. The mean threshold cycle value $(\mathrm{Ct})$ of each sample was normalized to the internal control, GAPDH, and the expression profiles were obtained by comparing normalized $\mathrm{Ct}$ value with the calibrator sample, in which the gene exhibited the lowest expression level. Each analysis was performed in triplicate. Quantification of each sample was calculated with the cycle threshold values and standard curve information using the Lightcycler 480 version 1.5.0 software.

\section{Peptide synthesis}

Nineteen synthetic linear peptides (Table 1) corresponding to chicken defensins, cathelicidins and NK-lysin, were synthesized and purified to $>95 \%$ purity grade through reverse-phase high-pressure liquid chromatography (Abclon, Seoul, Korea). Lyophilized peptide (1 mg each) was stored in desiccant at $-20{ }^{\circ} \mathrm{C}$ and dissolved in phosphate buffer ( $\mathrm{pH}$ 7.2) before use.

\section{Cell viability analysis of Escherichia coli after treatment with peptides}

Gram-negative bacteria, Escherichia coli ATCC 25922, were purchased from Korean Collection for Type
Culture and tested against each peptide. Cell viability analysis was carried out as previously reported [23]. Briefly, $6 \times 10^{6}$ colony-forming units (CFU)/ml bacteria suspensions $(90 \mu \mathrm{l})$ were placed into 96 well plates, followed by the addition of $10 \mu \mathrm{l}$ of serial diluted peptide (final $0,0.5,1,2.5,5 \mu \mathrm{M}$ ) in triplicate. After $2 \mathrm{~h}$ incubation at $37{ }^{\circ} \mathrm{C}$, equal volume of BacTiter-Glo ${ }^{\text {Tx }}$ Reagent (Promega) was added, and incubated for $5 \mathrm{~min}$ after which luminescence was measured with GloMax-Multi Detection System (Promega).

Detection of damaged E. coli membranes after treatment with synthetic cathelicidin peptides

To visualize E. coli membrane damage, $6.5 \times 10^{6} \mathrm{CFU}$ of $E$. coli were incubated with $5 \mu \mathrm{M}$ CATH1, CATH2, CATH3, or CATHB1 in $10 \mathrm{mM}$ phosphate buffer (pH 7.0), respectively, at $37{ }^{\circ} \mathrm{C}$ for $2 \mathrm{~h}$, and the membranes were observed by confocal laser scanning microscopy (Carl Zeiss, Oberkochen, Germany) after staining with the LIVE/DEAD BacLight bacterial viability kit (Invitrogen) according to the manufacturer's protocol.

\section{Scanning electron microscopic analysis}

To visualize membrane damages of E. coli, $6.5 \times$ $10^{6} \mathrm{CFU}$ of E. coli were incubated with $5 \mu \mathrm{M}$ CATH1, CATH2, CATH3, or CATHB1 peptides at $37{ }^{\circ} \mathrm{C}$ for

Table 1 Sequences and properties of peptides used in this study

\begin{tabular}{llllll}
\hline Name & Length (aa) & Composition & Mw (g/mol) & Net charge & Hydrophobic ratio \\
\hline AvBD1 & 40 & GRKSDCFRKSGFCAFLKCPSLTLISGKCSRFYLCCKRIWG & 4567.55 & 7.7 & 32 \\
AvBD2 & 39 & RDMLFCKGGSCHFGGCPSHLIKVGSCFGFRSCKWPWNA & 4324.14 & 3.9 & 36 \\
AvBD3 & 36 & TATQCRIRGGFCRVGSCRFPHIAIGKCATFISCCGR & 3877.62 & 5.8 & 33 \\
AvBD4 & 37 & RYHMQCGYRGTFCTPGKCPYGNAYLGLCRPKYSCCRW & 4341.08 & 5.8 & 24 \\
AvBD5 & 37 & GLPQDCERRGGFCSHKSCPPGIGRIGLCSKEDFCCRS & 4000.59 & 1.8 & 24 \\
AvBD6 & 38 & SPIHACRYQRGVCIPGPCRWPYYRVGSCGSGLKSCCVR & 4216.96 & 5.8 & 32 \\
AvBD7 & 37 & RPIDTCRLRNGLCFPGICRRPYYWIGTCNNGIGSCCAR & 4307.05 & 4.7 & 32 \\
AvBD8 & 41 & NNEAQCEQAGGICSKDHCFHLHTRAFGHCQRGVPCCRTVYD & 4593.16 & 0.1 & 24 \\
AvBD9 & 42 & ADTLACRQSMGSCSFVACRAPSVDIGTCRGGKLKCCKWAPSS & 4353.13 & 3.7 & 36 \\
AvBD10 & 42 & PDTVACRTQGNFCRAGACPPTFTISGQCHGGLLNCCAKIPAQ & 4309.01 & 1.8 & 38 \\
AvBD11 & 39 & RDTSRCVGYHGYCIRSKVCPKPFAAFGTCSWRQKTCCVD & 4431.14 & 4.8 & 28 \\
AvBD12 & 44 & PDSCNHDRGLCRVGNCNPGEYLAKYCFEPVILLCCKPLSPTPTKT & 4841.64 & 0.8 & 36 \\
AvBD13 & 37 & SDSQLCRNNHGHCRRLCFHMESWAGSCMNGRLRCCRF & 4373.03 & 4 & 24 \\
AvBD14 & 37 & DTVTCRKMKGKCSFLLCPFFKRSGTCYNGLAKCCRP & 4151 & 6.7 & 30 \\
CATH1 & 28 & PVRVKRVWPLVIRTVIAGYNLYRAIKKK & 3338.14 & 8 & 53 \\
CATB1 & 40 & PIRNWWIRIWEWLNGIRKRLRQRSPFYVRGHLNVTSTPQP & 5028.86 & 7.1 &
\end{tabular}

Molecular weight, net charge at $\mathrm{pH} 7$ and hydrophobic ratio were calculated using Peptide property calculator (http://www.innovagen.proteomics-tools) 
5 min. After incubation, the bacteria were fixed with $2 \%$ glutaraldehyde, washed, mounted, and damaged membranes were observed by a Field-Emission Scanning Electron Microscope (Carl Zeiss Inc.).

\section{Statistical analysis}

GraphPad prism software was used for cell viability data analyses and gene expression analysis and data were expressed as mean \pm SD. Statistical significance between groups or conditions was analysed by two-way or oneway ANOVA followed by Bonferroni's post hoc test unless stated otherwise. Differences were considered to be statistically significant when $p<0.05$.

\section{Results}

Tissue expression patterns

Quantitative RT-PCR was performed to examine the expression patterns of $C A T H, A v B D$ and $N K$-lysin genes in various chicken tissues. The chicken $A v B D$ gene family has a unique expression pattern. $c A v B D 1$ through 7 are predominantly expressed in bone marrow and weakly expressed in thymus. $A v B D 5$ is an exception with strong expression in the thymus. The other $A v B D s, A v B D 8$ through 10 and $A v B D 13$ are predominantly expressed in liver. $A v B D 11, A v B D 12$ and $A v B D 14$ are expressed in all tissues tested (Fig. 1). Chicken CATH1, -2 , and -3 are predominantly expressed in the bone marrow and to a lesser extent in bursa of Fabricius and thymus. CATHB1, however, showed abundant expression in bursa of Fabricius with low levels of expression in thymus and cecal tonsils. $N K$-lysin was predominantly expressed in spleen and in the duodenal loop, with lesser expression in thymus and bone marrow.

\section{Antimicrobial activity of chicken HDPs}

To address and compare the relative antibacterial activity of chicken HDPs, $14 A v B D, 4 C A T H$ and one NK-lysin peptide were synthesized and tested for antimicrobial

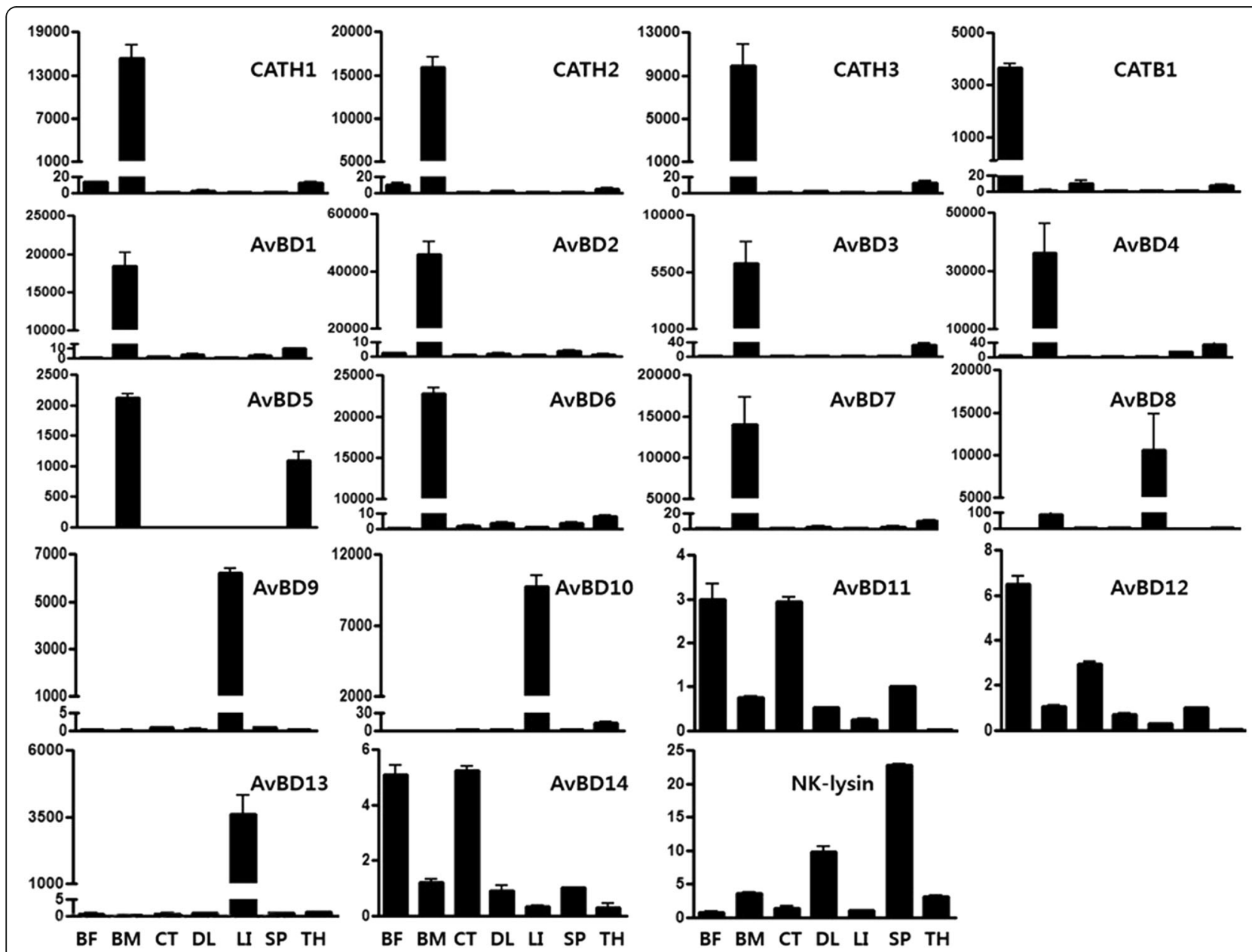

Fig. 1 Tissue expression of chicken HDPs. Quantitative RT-PCR analysis of CATH, AvBD and NK-lysin gene expression in chicken tissues. The relative abundance of mRNA was assessed by normalization and calibrated to glyceraldehyde 3-phosphate dehydrogenase (GAPDH). BF Bursa of Fabricius, $B M$ bone marrow, $C T$ cecal tonsil, $D L$ duodenal loop, $L /$ liver, SP spleen and $T H$ thymus 
activity as previously described [23]. Most of the tested HDPs showed strong antibacterial activity against E.coli (Fig. 2a), but 4 peptides AvBD5, -8, -10 and -12, showed very weak lytic activity at $5 \mu \mathrm{M}$. The majority of the peptides, however, killed more than $80 \%$ of E.coli under test conditions.

We selected peptides that exhibited strong antimicrobial activity at $5 \mathrm{uM}$ (less than $15 \%$ survival rate), and tested antibacterial activity with lower concentrations of peptide $(0.5,1,2.5$ and $5 \mu \mathrm{M})$. These HDPs killed bacteria in a dose-dependent manner. Most peptides produced less than $20 \%$ bacterial survival at low micromolar concentration $(2.5 \mu \mathrm{M})$ and AvBD4, AvBD6, AvBD7, CATH1, CATH2, CATH3 and cNK3 show very strong antibacterial activity at all concentration (Fig. 2b). These peptides killed $50 \%$ of bacteria at very low $(0.5 \mu \mathrm{M})$ micromolar concentration. AvBD4 and CATH3 displayed the strongest antibacterial effect among all tested chicken HDPs under test conditions. These results suggest that all functional peptides from chicken HDP had effective antibacterial activities under a broad range of peptide concentrations.
Membrane damage by synthetic peptides

To determine whether chicken HDPs altered the morphology and viability of $E$. coli, we used four cathelicidin peptides that have strong antimicrobial activity (Fig. 2). The damage to $E$. coli cell membranes after treatment with peptide was determined with confocal laser scanning microscopy. In the absence of peptide, most of the E. coli cells were stained green, indicating an intact membrane. In the presence of $5 \mu \mathrm{M}$ peptide treatment, the majority of $E$. coli cells were stained red, indicating membrane damage (Fig. 3). The membrane damage was greater with CATH2 or CATH3 than with CATH1 or CATHB1, consistent with dose-dependent cell killing data.

Scanning electron microscopy showed that untreated $E$. coli cells had normal, intact shape and uniform membrane surface (Fig. 4a and b). However, after treatment with each $\mathrm{CATH}$ an obvious difference in morphology was observed (Fig. $4 \mathrm{c}-\mathrm{j}$ ). The treated cells showed shrinkage, were rumpled, and lost their regularly arranged surface layer. The burst and crushed appearing cells were surrounded by debris. These findings suggest that cathelicidin destroy bacterial cells via membrane damage.

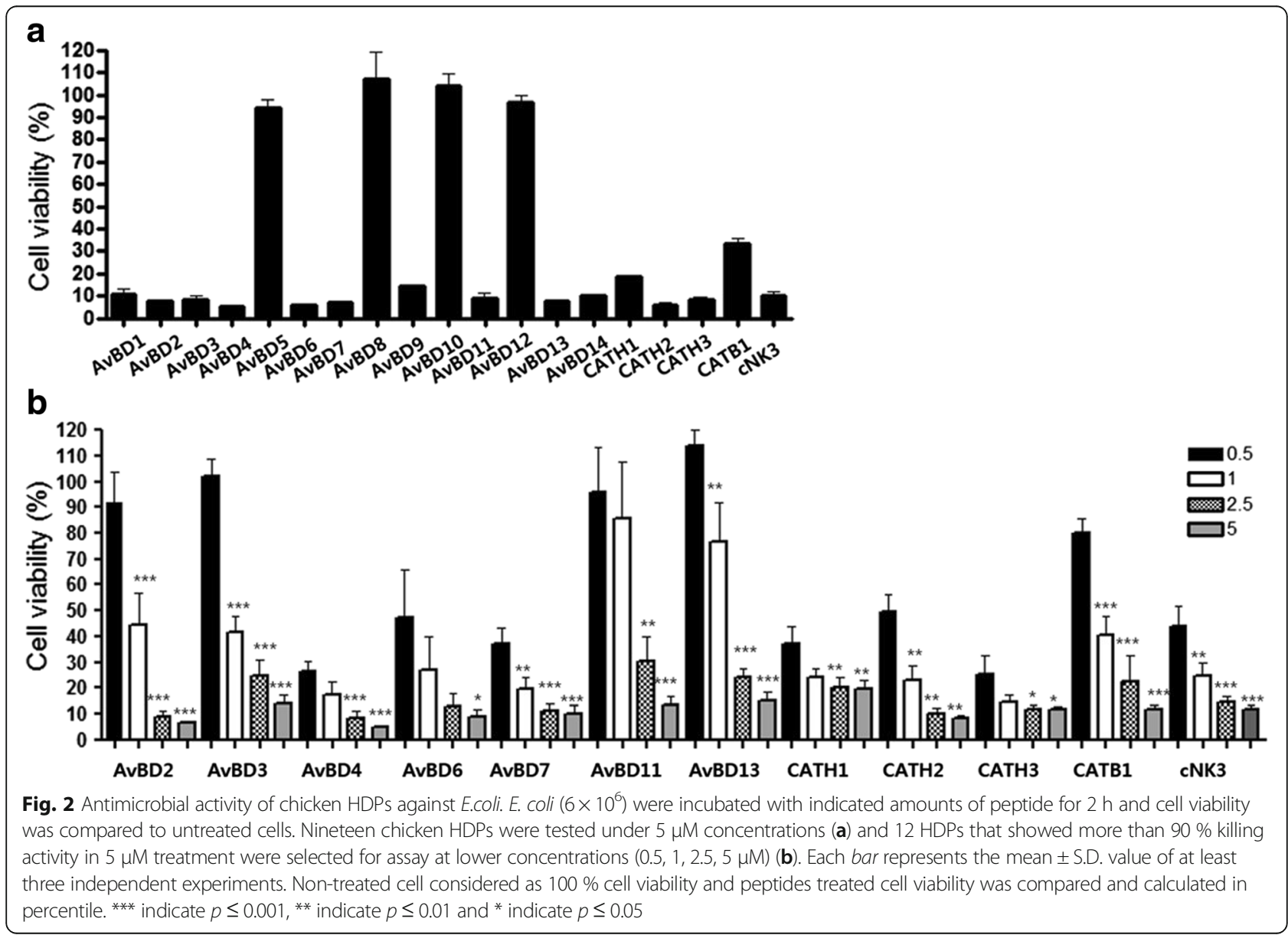




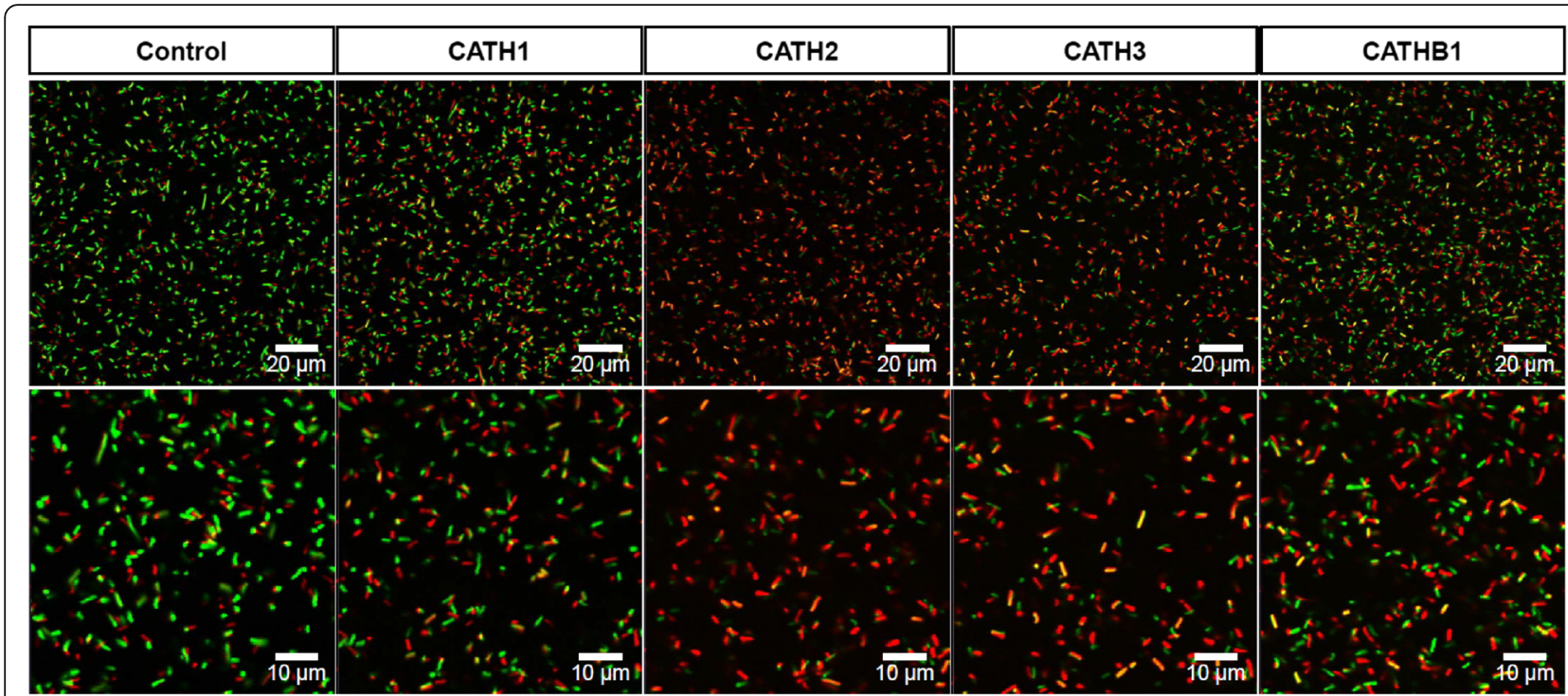

Fig. 3 Escherichia coli viability and membrane damage following treatment with cathelicidins. Membrane damage in E. coli treated with each synthetic cathelicidin peptide $(5 \mu \mathrm{M})$ was detected with a LIVE/DEAD BacLight Bacterial Viability Kit. Green fluorescence indicates live bacteria with intact membranes; red fluorescence indicates dead bacteria with damaged membranes. CATH1 cathelicidin1, CATH2 cathelicidin2, CATH3 cathelicidin3, and CATHB1 cathelicidinB1

\section{Discussion}

The aim of this study was to enhance understanding of tissue expression and antibacterial activity of chicken HDPs and to determine whether chickens can be a source of bioactive HDPs. As an initial step to understand HDPs, we examined their tissue expression profile, mainly in immune organs such as bursa of Fabricius, bone marrow, spleen and thymus. Cecal tonsil, duodenal loop and liver were also tested. Like their mammalian counterparts, avian cathelicidins and $\beta$-defensins are derived from bone marrow and/or epithelial cells. Chicken cathelicidins CATH1, -2 and -3 , are highly expressed in bone marrow with little to no expression in other tissues. In agreement with their myeloid origin, CATH1-3 mRNA has been found abundantly in the bone marrow [36]. On the other hand, chicken CATHB1 mRNA shows a more restricted expression pattern, with preferential expression in bursa of Fabricius.

$A v B D s$ expression is seen mainly in bone marrow for $A v B D 1$ through 7, which originate from myeloid cells, and in liver for $A v B D 8$ through 10 and -13. Other lymphoid tissues did not express $A v B D s$ in significant amounts. $A v B D 2$ and -4 expression is especially very weak and is limited to bone marrow and $A v B D 13$ is weakly detected in liver but hardly detected in other tissues even with increased PCR cycles relative to the others. These results are consistent with previous reports [36, 40]. Only $A v B D 5$ was strongly expressed in thymus. These results show that the tissue specific pattern varies across the defensin gene family with some members showing expression in all tested tissues, whereas the majority demonstrate more limited expression patterns which can be divided into three groups. Seven genes ( $A v B D 1$ through 7$)$ are predominantly expressed in bone marrow, four genes $(A v B D 8$ through 10 and 13) are restricted primarily to liver, and three $(A v B D 1,-12$ and -14$)$ are expressed in all tested tissues. $N K$-lysin showed strong expression in spleen with intermediate expression in bone marrow, intestine and thymus. Consistent with the role of cathelicidins, defensins and NK-lysin in the first line of host defense, abundant expression of these genes was detected in bursa and bone marrow. The transcriptional regulatory mechanism of these genes during development and under pathogen infection remains to be demonstrated.

The antibacterial efficacy of several defensins, cathelcidins and NK-lysin has been evaluated [2, 9, 23, 36, 40]. Like their mammalian counterparts, most chicken $A \nu B D$, CATHs and NK-lysin are capable of killing bacteria. Cuperus et al. reviewed antimicrobial activity of avian HDPs against selected pathogens [41]. Zhang and Sunkara also reviewed expression, antimicrobial and immunomodulatory activities of HDPs, but there are no direct, comprehensive comparisons of major HDPs [30]. Here, we synthesized 19 chicken HDPs and analyzed antibacterial activity against $E$. coli in a comprehensive direct comparison. These peptides differ in net charge from 0.1 to 10 and also vary in length from 28 to 44 amino acids. They also vary in expected hydrophobicity from 24 to $61 \%$ (Table 1 ). 

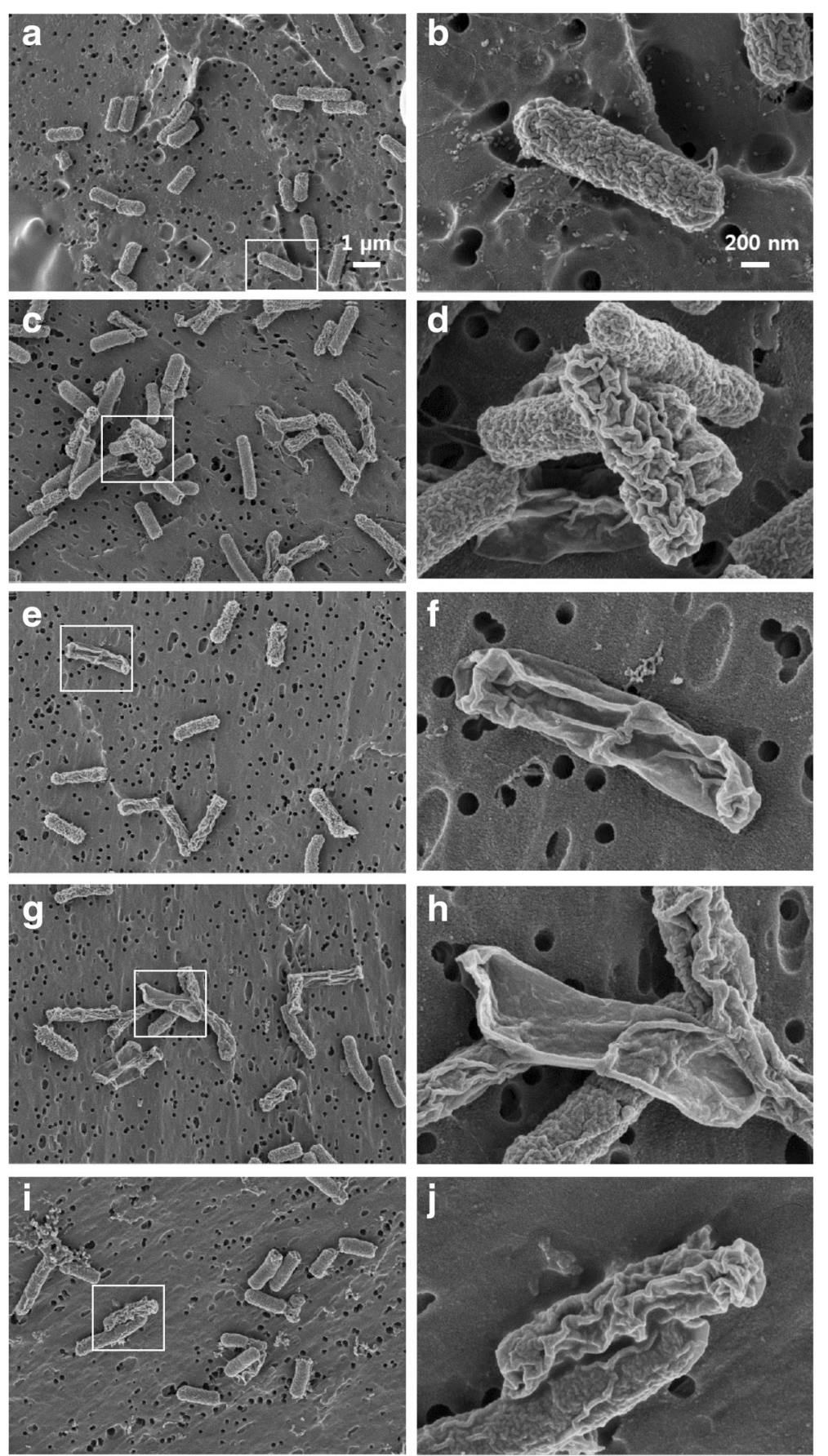

Fig. 4 Scanning electron micrographs of cathelicidin-induced cell membrane damage. Scanning electron micrographs of E. coli with no treatment (control, $\mathbf{a}$ and $\mathbf{b}$ ) or treated with cathelicidin 1 (CATH1, $\mathbf{c}$ and $\mathbf{d}$ ), cathelicidin2 (CATH2, e and $\mathbf{f}$ ), cathelicidin3 (CATH3, $\mathbf{g}$ and $\mathbf{h}$ ), or cathelicidinB1 (CATHB1, $\mathbf{i}$ and $\mathbf{j})$. The rectangle as shown in the large-scale image

Even though many tested chicken HDPs show varying efficiencies against pathogens, the majority kill bacteria at low concentration. AvBD5, -8, -10 and -12 show minimal killing activity among tested HDPs at less than $5 \mu \mathrm{M}$. This is consistent with the previous report that AvBD8 activity showed $27 \mu \mathrm{M}$ as lethal dose (LD) 50 against E.coli [42]. These four peptides have very low net charge from 0.1 to 1.8 . AvBD4 has $24 \%$ hydrophobicity with 5.8 net charge and can kill bacteria very well compared to AvBD5, -8 and -13 that have the same hydrophobicity but lower cationicity of 0.1 to 4. This suggests that low net charge results in 
inefficient antibacterial efficacy, even with a suitable hydrophobicity. However, AvBD3, -4 and -6 have the same net charge (5.8) and these peptides kill effectively over all tested concentrations, although BD4 has $33 \%$ hydrophobicity and the strongest activity among the three. Also, there is $6 \%$ gap in hydrophobicity between AvBD2 and cNK3, which share 3.9 net charge but demonstrate different activity. This result suggests that, hydrophobicity is an important factor in antibacterial activity of peptides. A structural effect cannot be ruled out but this result revealed that antimicrobial activity is strongly influenced by cationicity and hydrophobicity. Although, the $C A T H$ family has more overall cationicity and hydrophobicity than the $A \nu B D$ family, but this does not translate to higher antibacterial activity. The C-terminus of some of our synthesized peptides was abbreviated relative to natural peptides. We recognize that this could impact cationicity and hydrophobicity on antibacterial activity. This potential discrepancy should be clarified in future experiment.

In the present study, all four CATHs reduced E. coli cell viability and severely damaged $E$. coli cell membranes, with CATH2 and CATH3 showing the highest efficiency. In previous studies, the variable domains of CATH1, CATH2, and CATH3 were clearly discriminated, but the variable domain of CATHB1 was not identified [35, 36]. Here, we predicted the variable domain sequence of CATHB1 and demonstrated weak antibacterial activity compared to others.

Mechanisms for bacteria killing by $\beta$-defensin are thought to be similar to those of other cationic HDPs where positively charged residues interact with negatively charged membrane components, after which hydrophobic residues insert into the membrane, disrupting it and killing the cells $[43,44]$. It is generally accepted that increasing the hydrophobicity of the nonpolar face of the amphipathic $\alpha$-helical peptides will also increase the antimicrobial activity $[45,46]$. Increased cationicity also helps to enhance antibacterial activity [9]. Cationicity is important for killing bacteria [47], but simply increasing the net charge does not result in the improvement of antimicrobial potency [48]. Hydrophobicity also requires an optimal range to enhance antibacterial activity [45]. Disruption of structural integrity is another important factor for high efficiency in bacteria killing $[49,50]$. Our results indicate that the mode of antibiotic action of HDPs requires a balance between cationicity and hydrophobicity to optimize bacteria killing activity. But the relationships between two important factors, cationicity and hydrophobicity, and antimicrobial efficacy of HDPs remains to be determined. Antimicrobial efficacy of 19 peptides against E.coli in this study are consistent with the previous report [35]. Future study against other bacteria that cause bacterial disease in birds will help to improve our understanding of the role of these genes in immunity to bacteria in chickens.

\section{Conclusions}

Antimicrobial activity and differential tissue expression patterns of 19 chicken HDPs were analyzed. In summary, we confirmed that most of the HDPs showed antibacterial activity against E.coli and demonstrate their differential tissue expression pattern. These studies highlight the dose-dependent antimicrobial effects that were mediated by membrane damage and the importance of balance between cationicity and hydrophobicity. Gene expression of chicken HDPs are variable and the AvBD gene family can be divided into two functional expressional groups.

\section{Additional file}

Additional file 1: Table S1. Primers used in this study. (DOCX $14 \mathrm{~kb}$ )

Abbreviations

AMP: Antimicrobial peptide; HDPs: Host defense peptides

\section{Acknowledgements}

None.

\section{Funding}

This research was supported by Grant PJ008142 from the Next-Generation BioGreen 21 program, Rural Development Administration and WCU (World Class University) program (R31-10056) through the National Research

Foundation of Korea funded by the Ministry of Education, Science and Technology.

Availability of data and materials

All data supporting the results of this study are included within the article.

Authors' contributions

Conceived and designed the experiments: MOL HJ JYH JEW. Performed the experiments: MOL and HJ. Analyzed the data: MOL HJ DR SY SJL. Wrote the paper: MOL HJ JEW. Provide sample: SJL. All authors amended the draft and approved the final manuscript.

Competing interests

The authors declare that they have no competing interests.

Consent for publication

Not applicable.

Ethics approval and consent to participate

Ethical and humane treatment of animals used in this study was proposed to and approved by the Institutional of Animal Care and Use Committee at lowa State University in accordance with U.S. Government principles on ethical utilization and care of vertebrate animals as described in the Animal Welfare Act (7 U.S.C. 2131 et. Seq.).

\section{Author details}

'WCU Biomodulation Major, Department of Agricultural Biotechnology, Seoul National University, Seoul, Korea. ${ }^{2}$ Department of Veterinary Pathobiology, Texas A \& M University, College Station, TX, USA. ${ }^{3}$ College of Pharmacy, Dankook University, Cheonan, Korea. ${ }^{4}$ Department of Animal Science, lowa State University, Ames, IA, USA. 


\section{Received: 2 October 2015 Accepted: 11 October 2016}

Published online: 13 October 2016

\section{References}

1. Wigley P. Immunity to bacterial infection in the chicken. Dev Comp Immunol. 2013:41:413-7.

2. Lehrer RI, Ganz T. Defensins of vertebrate animals. Curr Opin Immunol. 2002; 14:96-102.

3. Zanetti M. The role of cathelicidins in the innate host defenses of mammals. Curr Issues Mol Biol. 2005;7:179-96.

4. Tossi A, Sandri L, Giangaspero A. Amphipathic, alpha-helical antimicrobial peptides. Biopolymers. 2000;55:4-30.

5. Broekaert WF, Terras FR, Cammue BP, Osborn RW. Plant defensins: novel antimicrobial peptides as components of the host defense system. Plant Physiol. 1995:108:1353-8.

6. Hancock RE, Sahl HG. Antimicrobial and host-defense peptides as new antiinfective therapeutic strategies. Nat Biotechnol. 2006;24:1551-7.

7. Zasloff M. Antimicrobial peptides of multicellular organisms. Nature. 2002; 415:389-95.

8. Pasupuleti M, Schmidtchen A, Malmsten M. Antimicrobial peptides: key components of the innate immune system. Crit Rev Biotechnol. 2012;32: $143-71$.

9. Lee MO, Kim EH, Jang HJ, Park MN, Woo HJ, Han JY, Womack JE. Effects of a single nucleotide polymorphism in the chicken NK-lysin gene on antimicrobial activity and cytotoxicity of cancer cells. Proc Natl Acad Sci U S A. 2012:109:12087-92.

10. Boman HG. Peptide antibiotics and their role in innate immunity. Annu Rev Immunol. 1995;13:61-92

11. Mangoni ML, Rinaldi AC, Di Giulio A, Mignogna G, Bozzi A, Barra D, Simmaco M. Structure-function relationships of temporins, small antimicrobial peptides from amphibian skin. Eur J Biochem. 2000;267:1447-54.

12. He LH, Lemasters JJ. Regulated and unregulated mitochondrial permeability transition pores: a new paradigm of pore structure and function? Febs Letters. 2002;512:1-7.

13. Matsuzaki K. Why and how are peptide-lipid interactions utilized for selfdefense? Magainins and tachyplesins as archetypes. Biochim Biophys Acta. 1999;1462:1-10.

14. Epand RM, Vogel HJ. Diversity of antimicrobial peptides and their mechanisms of action. Biochimica Et Biophysica Acta-Biomembranes. 1999; 1462:11-28.

15. Ostberg N, Kaznessis Y. Protegrin structure-activity relationships: using homology models of synthetic sequences to determine structural characteristics important for activity. Peptides. 2005;26:197-206.

16. Bu X, Wu X, Xie G, Guo Z. Synthesis of tyrocidine A and its analogues by spontaneous cyclization in aqueous solution. Org Lett. 2002;4:2893-5.

17. Ovchinnikova TV, Aleshina GM, Balandin SV, Krasnosdembskaya AD, Markelov ML, Frolova El, Leonova YF, Tagaev AA, Krasnodembsky EG, Kokryakov VN. Purification and primary structure of two isoforms of arenicin, a novel antimicrobial peptide from marine polychaeta Arenicola marina. Febs Letters. 2004;577:209-14.

18. Imamura T, Yasuda M, Kusano H, Nakashita H, Ohno Y, Kamakura T, Taguchi S, Shimada $\mathrm{H}$. Acquired resistance to the rice blast in transgenic rice accumulating the antimicrobial peptide thanatin. Transgenic Res. 2010;19:415-24.

19. Rahnamaeian $M$, Langen $G$, Imani J, Khalifa W, Altincicek B, von Wettstein D, Kogel KH, Vilcinskas A. Insect peptide metchnikowin confers on barley a selective capacity for resistance to fungal ascomycetes pathogens. J Exp Bot 2009:60:4105-14.

20. Wu M, Hancock RE. Improved derivatives of bactenecin, a cyclic dodecameric antimicrobial cationic peptide. Antimicrob Agents Chemother. 1999:43:1274-6.

21. van Dijk A, Molhoek EM, Veldhuizen EJ, Bokhoven JL, Wagendorp E, Bikker F, Haagsman HP. Identification of chicken cathelicidin-2 core elements involved in antibacterial and immunomodulatory activities. Mol Immunol. 2009:46:2465-73.

22. Xiao Y, Herrera Al, Bommineni YR, Soulages $J$, Prakash O, Zhang G. The central kink region of fowlicidin-2, an alpha-helical host defense peptide, is critically involved in bacterial killing and endotoxin neutralization. J Innate Immun. 2009:1:268-80.

23. Lee MO, Jang HJ, Han JY, Womack JE. Chicken NK-lysin is an alpha-helical cationic peptide that exerts its antibacterial activity through damage of bacterial cell membranes. Poult Sci. 2014;93:864-70.
24. Hancock RE, Nijnik A, Philpott DJ. Modulating immunity as a therapy for bacterial infections. Nat Rev Microbiol. 2012;10:243-54.

25. Choi KY, Chow LN, Mookherjee N. Cationic host defence peptides: multifaceted role in immune modulation and inflammation. J Innate Immun. 2012:4:361-70.

26. Semple F, Dorin JR. beta-Defensins: multifunctional modulators of infection, inflammation and more? J Innate Immun. 2012;4:337-48.

27. Selsted ME, Ouellette AJ. Mammalian defensins in the antimicrobial immune response. Nat Immunol. 2005;6:551-7.

28. Zanetti M. Cathelicidins, multifunctional peptides of the innate immunity. J Leukoc Biol. 2004;75:39-48.

29. Schutte BC, McCray Jr PB. [beta]-defensins in lung host defense. Annu Rev Physiol. 2002;64:709-48.

30. Zhang G, Sunkara LT. Avian antimicrobial host defense peptides: from biology to therapeutic applications. Pharmaceuticals (Basel). 2014;7:220-47.

31. Evans EW, Beach GG, Wunderlich J, Harmon BG. Isolation of antimicrobial peptides from avian heterophils. J Leukoc Biol. 1994;56:661-5.

32. Gong D, Wilson PW, Bain MM, McDade K, Kalina J, Herve-Grepinet V, Nys Y, Dunn IC. Gallin; an antimicrobial peptide member of a new avian defensin family, the ovodefensins, has been subject to recent gene duplication. BMC Immunol. 2010;11:12

33. Lynn DJ, Higgs R, Lloyd AT, O'Farrelly C, Herve-Grepinet $V$, Nys Y, Brinkman FS, Yu PL, Soulier A, Kaiser P, et al. Avian beta-defensin nomenclature: a community proposed update. Immunol Lett. 2007;110:86-9.

34. Xiao Y, Hughes AL, Ando J, Matsuda Y, Cheng JF, Skinner-Noble D, Zhang G. A genome-wide screen identifies a single beta-defensin gene cluster in the chicken: implications for the origin and evolution of mammalian defensins. BMC Genomics. 2004;5:56.

35. Xiao Y, Cai Y, Bommineni YR, Fernando SC, Prakash O, Gilliland SE, Zhang G. Identification and functional characterization of three chicken cathelicidins with potent antimicrobial activity. J Biol Chem. 2006;281:2858-67.

36. Goitsuka R, Chen CL, Benyon L, Asano Y, Kitamura D, Cooper MD. Chicken cathelicidin-B1, an antimicrobial guardian at the mucosal M cell gateway. Proc Natl Acad Sci U S A. 2007:104:15063-8.

37. Lee MO, Yang E, Morisson M, Vignal A, Huang YZ, Cheng HH, Muir WM, Lamont SJ, Lillehoj HS, Lee SH, et al. Mapping and genotypic analysis of the NK-lysin gene in chicken. Genet Sel Evol. 2014;46:43

38. Hasenstein JR, Lamont SJ. Chicken gallinacin gene cluster associated with Salmonella response in advanced intercross line. Avian Dis. 2007:51:561-7.

39. McCormack WT, Tjoelker LW, Thompson CB. Avian B-cell development: generation of an immunoglobulin repertoire by gene conversion. Annu Rev Immunol. 1991:9:219-41.

40. Harwig SS, Swiderek KM, Kokryakov VN, Tan L, Lee TD, Panyutich EA, Aleshina GM, Shamova OV, Lehrer RI. Gallinacins: cysteine-rich antimicrobial peptides of chicken leukocytes. FEBS Lett. 1994;342:281-5.

41. Cuperus T, Coorens M, van Dijk A, Haagsman HP. Avian host defense peptides. Dev Comp Immunol. 2013:41:352-69.

42. Higgs R, Lynn DJ, Cahalane S, Alana I, Hewage CM, James T, Lloyd AT, O'Farrelly C. Modification of chicken avian beta-defensin-8 at positively selected amino acid sites enhances specific antimicrobial activity. Immunogenetics. 2007:59:573-80.

43. Brogden KA. Antimicrobial peptides: pore formers or metabolic inhibitors in bacteria? Nat Rev Microbiol. 2005;3:238-50.

44. Powers JP, Hancock RE. The relationship between peptide structure and antibacterial activity. Peptides. 2003;24:1681-91.

45. Chen Y, Guarnieri MT, Vasil Al, Vasil ML, Mant CT, Hodges RS. Role of peptide hydrophobicity in the mechanism of action of alpha-helical antimicrobial peptides. Antimicrob Agents Chemother. 2007:51:1398-406.

46. Wieprecht T, Dathe M, Beyermann M, Krause E, Maloy WL, MacDonald DL, Bienert M. Peptide hydrophobicity controls the activity and selectivity of magainin 2 amide in interaction with membranes. Biochemistry. 1997;36:6124-32.

47. Ma D, Lin L, Zhang K, Han Z, Shao Y, Liu X, Liu S. Three novel Anas platyrhynchos avian beta-defensins, upregulated by duck hepatitis virus, with antibacterial and antiviral activities. Mol Immunol. 2011;49:84-96.

48. Dong N, Ma QQ, Shan AS, Lv YF, Hu W, Gu Y, Li YZ. Novel design of short antimicrobial peptides derived from the bactericidal domain of avian betadefensin-4. Protein Pept Lett. 2012;19:1212-9.

49. Takahashi D, Shukla SK, Prakash O, Zhang G. Structural determinants of host defense peptides for antimicrobial activity and target cell selectivity. Biochimie. 2010;92:1236-41.

50. Taylor K, Barran PE, Dorin JR. Structure-activity relationships in beta-defensin peptides. Biopolymers. 2008;90:1-7. 